Zbl Arbeitsmed 2020 $\cdot 70: 215-221$ https://doi.org/10.1007/s40664-020-00392-9 Online publiziert: 12. März 2020

(c) Der/die Autor(en) 2020

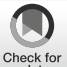

Madeleine Dulon' - Dana Wendeler ${ }^{2}$ - Claudia Westermann ${ }^{3} \cdot$ Albert Nienhaus $^{2,3}$

${ }^{1}$ Abteilung Arbeitsmedizin, Gefahrstoffe, Gesundheitswissenschaften, Berufsgenossenschaft für Gesundheitsdienst und Wohlfahrtspflege, Hamburg, Deutschland

${ }^{2}$ Berufsgenossenschaft für Gesundheitsdienst und Wohlfahrtspflege, Hamburg, Deutschland

${ }^{3}$ Competenzzentrum Epidemiologie und Versorgungsforschung bei Pflegeberufen (CVcare), Universitätsklinikum Hamburg-Eppendorf, Hamburg, Deutschland

\title{
Arbeitsunfälle und Berufskrankheiten bei Beschäftigten in Küchenbetrieben in Gesundheitsdienst und Wohlfahrtspflege
}

(BGW) ist der gesetzliche Unfallversicherungsträger (UV-Träger) für nichtstaatliche Einrichtungen im Gesundheitsdienst und in der Wohlfahrtspflege. Für Beschäftigte in Großküchen ist die BGW der zuständige UV-Träger, wenn das Unternehmen, bei dem sie angestellt sind, bei ihr versichert ist. Die BGW verzeichnete im Jahr 2018 rund 15.500 Versicherungsfälle, die Küchenhilfen und Köche betrafen, für die Entschädigungsleistungen in Höhe von 14,7 Mio. $€$ gezahlt wurden (interne Mitteilung der Reha-Statistik vom 17.09.2019). Das entsprach einem Anteil von 2,5\% an allen Entschädigungsleistungen der BGW in dem Jahr. Es handelt sich somit um eine vergleichsweise kleine Versichertengruppe, die gegenüber größeren Berufsgruppen häufig unbeachtet bleibt. Die Anzahl der Versicherungsfälle und die Höhe der Entschädigungsleistungen lassen eine differenzierte Analyse der Unfälle und Berufskrankheiten (BK) bei Beschäftigten in Küchenbetrieben als angemessen erscheinen. Ziel dieser Arbeit ist es, das Unfall- und BK-Geschehen getrennt für Köche und Küchenhilfen in Routinedaten der BGW zu analysieren.

\section{Methode}

Die vorliegende Sekundärdatenanalyse basiert auf personenbezogenen Routinedaten der BGW zu Arbeitsunfällen und BK-Fällen, die zwischen 2008 und 2017 gemeldet wurden. Es handelt sich um eine Querschnittstudie, für die eine Versichertenstichprobe gezogen wurde aus den drei Berufsgruppen Koch/Köchin, Küchenhilfe und Kantinenhilfe (ISCOSchlüssel 51229, 51211, 51212) und einer der vier Branchen (Krankenhaus/Klinik, Altenpflegeheim, ambulanter Dienst, Beratung sowie berufliche Rehabilitation inklusive Werkstätten für Menschen mit Behinderung). Die Selektion ergab insgesamt 87.862 meldepflichtige und nichtmeldepflichtige Arbeitsunfälle und 2340 Fälle mit einer meldepflichtigen BK-Verdachtsanzeige.

Für die Auswertung der Arbeitsunfälle wurden zwei weitere Einschlusskriterien festgelegt: Der Unfall hat bei der betrieblichen Tätigkeit am Arbeitsplatz stattgefunden und $\mathrm{zu}$ einer Arbeitsunfähigkeit von mindestens 3 Tagen geführt (sog. meldepflichtige Arbeitsunfälle; $n=40.703)$. Arbeitsunfälle im Straßenverkehr und Wegeunfälle sind nicht Gegenstand dieser Arbeit. Bei der Registrierung der Unfallmeldungen wer-

Die Berufsgenossenschaft für $\mathrm{Ge}$ sundheitsdienst und Wohlfahrtspflege 


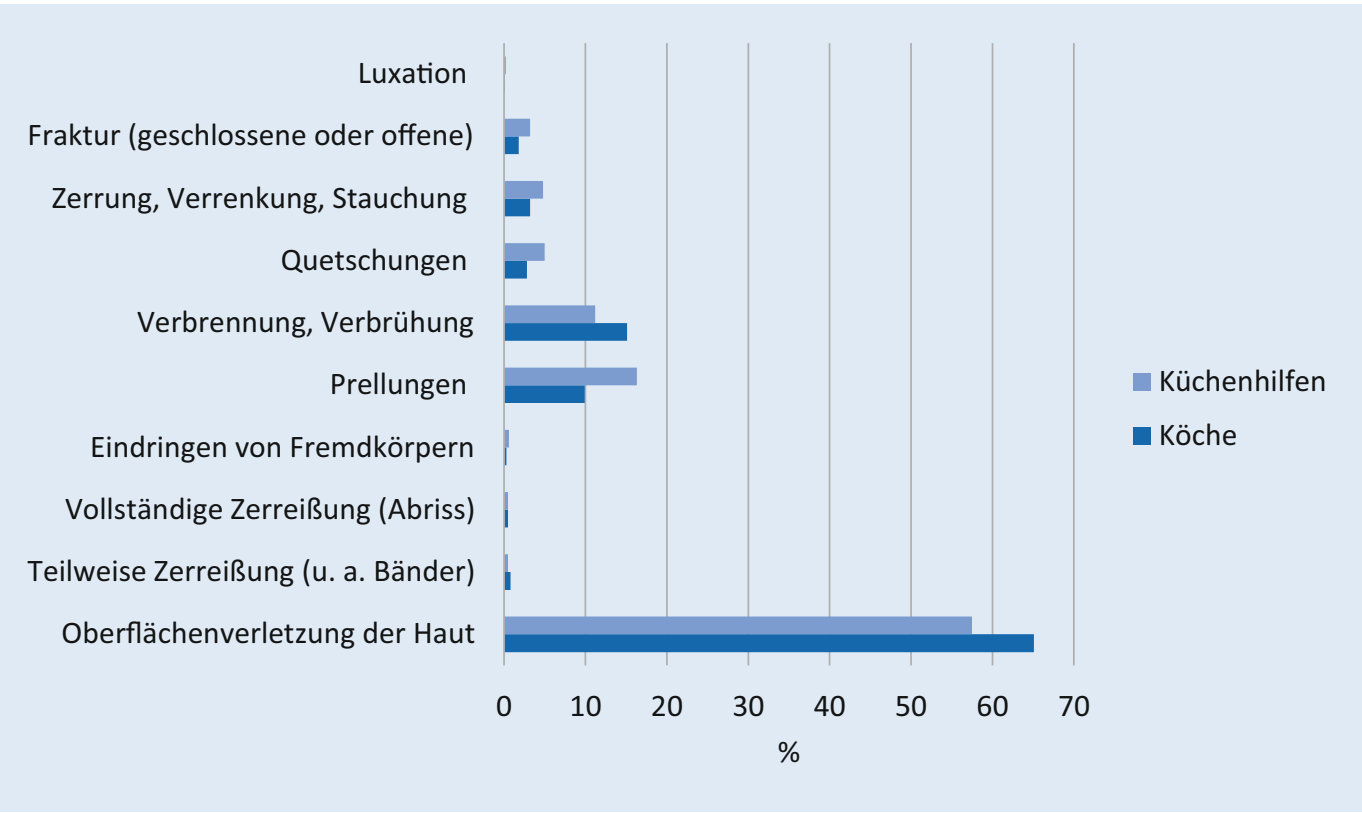

Abb. $1<$ Art der Verletzung im Bereich der Hände und Finger nach Beruf - meldepflichtige Arbeitsunfälle der Berufsgenossenschaft für Gesundheitsdienst und Wohlfahrtspflege (BGW) für 2008 bis 2017 den die Tatbestandsmerkmale, die für die Unfallstatistik erforderlich sind sowie Angaben zu Verletzungsart, der am schwersten betroffenen Stelle der Körperschädigung sowie Angaben zum Unfallhergang mittels elektronischem Datenverarbeitungsprogramm erfasst. Die differenzierte Dokumentation des Unfallhergangs wird wegen der großen Anzahl der zu verschlüsselnden Merkmale nur für $6,7 \%$ der meldepflichtigen Unfälle vorgenommen. Diese sog. „7-\%-Stichprobe" rekrutiert sich aus der Gesamtzahl der meldepflichtigen Unfälle, deren Geburtstag auf einen 10. oder 11. eines Monats fällt. Zur Analyse des Unfallhergangs wird die 7-\%-Stichprobe verwendet und daraus die folgenden Merkmale: die Begleitumstände unmittelbar vor dem Unfall (spezifische Tätigkeit), das letzte vom normalen Ablauf abweichende Ereignis (sog. Abweichung), die Art und Weise, wie das Opfer geschädigt wurde (Kontakt), und der unfallverursachende Gegenstand. Eine Hochrechnung der Stichprobenwerte wird nicht vorgenommen.

Anzeigen auf Verdacht einer BK durchlaufen ein Feststellungsverfahren mit einer versicherungsrechtlichen Entscheidung über Bestätigung oder Ablehnung des BK-Verdachts. Als BKauslösender Gegenstand werden Angaben zur gefährdenden Einwirkung erfasst, die in der Arbeitsanamnese erhoben werden und Bestandteil der versi- cherungsrechtlichen Entscheidung sind. Für die hier betrachtete Zielpopulation wurden in dem Bezugszeitraum 2330 BK-Fälle entschieden. Die Auswertung der gefährdenden Einwirkung erfolgte für eine Teilstichprobe aus entschiedenen Fällen mit bestätigtem BK-Verdacht (1820 von 2330).

Die Datenauswertung erfolgte deskriptiv-statistisch unter Angabe der absoluten und relativen Häufigkeiten getrennt nach Berufsgruppe. Kantinenhilfen, auf die ein Anteil von 2,8\% der Fälle entfiel, wurden der Gruppe der Küchenhilfen zugeordnet. Unterschiede zwischen den beiden Berufsgruppen wurden mittels Chi-Quadrat-Test oder exaktem Fisher-Test auf Signifikanz getestet. Das Signifikanzniveau wurde auf $p<0,01$ festgelegt. Die Analysen wurden mit dem Statistikprogramm IBMSPSS 25 (IBM Corp., Armonk, NY, USA) durchgeführt.

\section{Ergebnisse}

\section{Beschreibung der Stichproben zu Arbeitsunfällen und BK-Fällen}

Beide Stichproben waren hinsichtlich der soziodemografischen Merkmale vergleichbar. In beiden Stichproben waren rund 70,0\% als Küchenhilfe und 30,0\% als Koch/Köchin tätig. In der Gruppe der Küchenhilfen waren $84 \%$ weiblich; in der Gruppe der Köche lag dieser Anteil bei 45,0\%. Bei Köchen war im Vergleich zu Küchenhilfen der Anteil der unter 30-Jährigen größer ( $40 \%$ vs. $19 \%$ ) und der Anteil der über 50-Jährigen kleiner (23\% vs. $38 \%$ ). Die Arbeitsplätze beider Berufsgruppen befanden sich jeweils zu rund einem Drittel im Pflege- oder Klinikbereich.

\section{Arbeitsunfälle}

Beim Schweregrad der Arbeitsunfälle $(n=40.703)$ handelte es sich in beiden Berufsgruppen bei rund $70 \%$ um Bagatellfälle ohne längere Arbeitsunfähigkeits- oder Behandlungszeiten. Mittelschwere Unfälle mit weniger als sechs Wochen Arbeitsunfähigkeitszeiten und kürzeren Behandlungszeiten machten knapp ein Viertel der Unfälle aus, schwere Arbeitsunfälle mit längeren Arbeitsunfähigkeits- und Behandlungszeiten sowie eventuell bleibenden Körperschäden rund $4 \%$. Die Verletzungen betrafen vor allem die oberen Extremitäten. Am häufigsten war der Bereich um Hand, Finger und Handgelenk mit knapp 60,0\% und am zweithäufigsten der Bereich um Fuß, Zehen und Fußgelenk mit 11,5\% betroffen. Im Bereich der Hände machten oberflächliche Verletzungen der Haut rund $60 \%$ aus (- Abb. 1). An zweiter und dritter Stelle 


\section{Arbeitsunfälle und Berufskrankheiten bei Beschäftigten in Küchenbetrieben in Gesundheitsdienst und Wohlfahrtspflege}

\section{Zusammenfassung}

Hintergrund. Für Beschäftigte in Küchenbetrieben liegen nur wenige Daten zum Arbeitsunfall- und Berufskrankheitengeschehen für Deutschland vor. Für eine differenzierte Analyse wurden Routinedaten der Berufsgenossenschaft für Gesundheitsdienst- und Wohlfahrtspflege (BGW) genutzt.

Methoden. Eingeschlossen wurden Versicherte, die als Küchenhilfe oder Koch tätig waren und für die ein Arbeitsunfall oder eine Verdachtsanzeige auf eine Berufskrankheit (BK) bei der BGW zwischen 2008 und 2017 gemeldet wurde. Die Ergebnisse wurden für die Berufsgruppen deskriptiv beschrieben und Gruppenunterschiede mittels Chi-QuadratTest überprüft.
Ergebnisse. Rund 70\% der Unfälle und BKAnzeigen stammten von Küchenhilfen, 30\% von Köchen. Küchenhilfen verletzten sich häufiger bei Stolperunfällen und beim manuellen Transport von Gegenständen als Köche. Fast die Hälfte der Unfälle von Köchen wurde durch die Handhabung von Messern verursacht und knapp $17 \%$ durch Verbrennungen. BKVerdachtsanzeigen auf eine Hauterkrankung machten in beiden Gruppen jeweils über $80 \%$ aus. Bandscheibenbedingte Erkrankungen der Lendenwirbelsäule hatten einen Anteil von $5 \%$ und obstruktive Atemwegserkrankungen von $3 \%$. Der BK-Verdacht wurde in $78 \%$ bestätigt, in den meisten Fällen handelte es sich um Hauterkrankungen, ausgelöst durch Feuchtarbeit, Desinfektions- oder Reinigungsmittel.

Diskussion. Schnittverletzungen und Stolperunfälle werden auch von anderen Autoren als Unfallschwerpunkte in Küchenbetrieben beschrieben. Das BK-Geschehen wurde überwiegend durch Hauterkrankungen bestimmt, das in diesem Ausmaß von anderen Autoren nicht berichtet wird. Berichtet werden vielmehr hohe Prävalenzen für muskuloskeletale Beschwerden im Bereich von Schulter und Hand, für die sich in den hier vorliegenden Daten keine Belege fanden.

\section{Schlüsselwörter}

Küchenpersonal · Arbeitsunfall · Berufskrankheit $\cdot$ Unfallversicherung $\cdot$ Routinedaten

\section{Work-related injuries and occupational diseases among kitchen workers in healthcare services and social welfare}

\section{Abstract}

Background. Only few data are available on work-related accidents and occupational diseases for kitchen workers in Germany. Routine data based on claims data from the Occupational Accident Insurance for Healthcare and Welfare Services (BGW) were used for a differentiated analysis.

Methods. The analysis included BGW claims data between 2008 and 2017 for kitchen helpers and cooks with notification of a workrelated accident or a suspected occupational disease. Descriptive statistics were used for occupational groups and group differences were evaluated using a $x^{2}$-test.

Results. Around $70 \%$ of the accidents and notifications of suspected occupational diseases were from kitchen helpers and $30 \%$ from cooks. Kitchen helpers were more often injured by stumbling accidents and in the manual transport of objects than cooks. Almost half the accidents suffered by cooks were associated with handling knives and almost $17 \%$ with burns. In both groups more than $80 \%$ of the suspected occupational diseases were skin diseases and $5 \%$ were diseases associated with lumbar disc problems caused by the lifting and carrying heavy loads. Just under $3 \%$ were obstructive airway diseases. The occupational disease was confirmed in $78 \%$ of cases, which were mostly skin diseases caused by working with fluids, disinfectants or cleaning agents.
Discussion. Incision wounds and stumbling accidents are also described by other authors as the most common accidents among kitchen workers. Most occupational diseases among kitchen workers in health services were skin diseases but similar prevalence rates have not been reported by other authors. In contrast, a high prevalence of musculoskeletal symptoms in the shoulders or hands have been reported for which no evidence was found in the present data.

Keywords

Kitchen worker - Occupational injury . Occupational disease - Accident insurance . Claims data folgten Prellungen bzw. Verbrennungen und Verbrühungen.

\section{Unfallhergang}

Die Angaben zum Unfallhergang aus der 7-\%-Stichprobe $(n=2404)$ zeigen, dass es sich bei der spezifischen Tätigkeit, die die verletzte Person zum Zeitpunkt des Unfalls ausübte, bei rund einem Drittel aller Fälle um die manuelle Handhabung von Gegenständen handelte (•Tab. 1).
Bei den Umständen, die dem Unfall vorausgingen, waren $82 \%$ aller Fälle auf zwei Muster zurückzuführen: auf den Verlust der Kontrolle über eine aktiv gesteuerte Aktion - z. B. beim Umgang mit handgeführten Gegenständen - oder auf eine die verletzte Person überlastende Körperbewegung. Für den Verlust der Kontrolle über den eigenen Körper waren Stolpern und Ausrutschen die häufigsten Ursachen. Fehlende Aufmerksamkeit bei Bewegungen führte in knapp $28 \% \mathrm{zu}$
Verletzungen. Ein Materialschaden war für jeden sechsten Unfall verantwortlich. Bei der Art und Weise, wie die verletzte Person geschädigt wurde, bildeten Unfälle beim Hantieren mit scharfen oder spitzen Gegenständen die größte Gruppe, wobei es sich in den meisten Fällen um Handwerkzeuge zum Schneiden handelte. Haushaltsgeräte waren an jedem 5. Unfall beteiligt. Eine besondere Gruppe stellen diejenigen Unfälle dar, bei denen es zu Bedrohungen oder Übergriffen 
Tab. 1 Merkmale zum Unfallhergang bei Beschäftigten in Küchenbetrieben getrennt nach Berufsgruppe ${ }^{\mathrm{a}}$ - Daten der Berufsgenossenschaft für Gesundheitsdienst und Wohlfahrtspflege (BGW) für 2008 bis 2017

Merkmale zum Unfallhergang

\section{Berufsgruppe}

Küchenhilfen Köche

$n=1668 \quad n=736$

$\%^{b}$

Spezifische Tätigkeit (gruppiert)

Manuelle Handhabung von Gegenständen

Darunter:

Etwas in die Hand nehmen, ergreifen, halten

33,5

$\%^{\mathrm{b}}$

Gehen, Laufen, Hinauf- und Hinabsteigen

18,5

27,8

17,1

24,5

Arbeit mit nicht kraftbetriebenen Handwerkzeugen

22,5

34,9

26,3

Transport von Hand (z. B. hochheben, schieben,

6,9

3,5

5,9

ziehen)

Sonstige

9,3

6,5

8,4

Umstände, die dem Unfall vorausgingen (Abweichung vom normalen Ablauf)

Verlust der Kontrolle

Darunter:

Über handgeführte Gegenstände

$40,8 \quad 44,3$

41,9

Über eigenen Körper (Stolpern, Ausrutschen)

21,6

33,5

25,3

Körperbewegung

Darunter:

Mit starker körperliche Belastung (z. B. beim Heben, Tragen, Ziehen, Schieben)

Ohne körperliche Anstrengung durch unachtsame Bewegung (z. B. an etwas stoßen; auf etwas treten)

Materialschaden (Brechen von Feststoffen, Überlaufen/Spritzen von flüssigen Stoffen, Verdampfen)

Bedrohung, körperliche Gewalt

Sonstige

Kontakt

Mechanische Einflüsse

Darunter:

Kontakt mit scharfen oder spitzen Gegenständen

Aufprallen auf/gegen ortsfeste Gegenstände

Kontakt mit offenem Feuer oder heißen Gegenstän-

den

Akute körperliche Überlastung des Bewegungsappa-

rats

Übergriffe/Angriffe durch Menschen

Sonstige

Unfallverursachender Gegenstand

Handwerkzeuge zum Schneiden (u. a. Messer)

Bauliche Einrichtungen (Fenster, Türen, Treppen)

Darunter:

(Fuß-)Böden zu ebener Erde

Haushaltsgegenstände, -geräte

Sonstige

${ }^{a}$ \% \%-Stichprobe zu meldepflichtigen Arbeitsunfällen

${ }^{b}$ Bezugsgröße für Prozentzahlen ist die Summe der jeweiligen Spalte

'Ausgewertet wurde jeweils die erste von vier möglichen Nennungen in Form von Gewalteinwirkungen durch andere Menschen gekommen ist (0,7\%). In 16 der 17 Fälle, die hierunter fielen, waren Küchenhilfen betroffen.

Köche verletzten sich häufiger als Küchenhilfen beim Hantieren mit nichtkraftbetriebenen Handwerkzeugen, durch Verlust der Kontrolle über einen handgeführten Gegenstand und durch Verbrennungen oder Verbrühungen an offenem Feuer oder heißen Gegenständen. Durch den Umgang mit scharfen oder spitzen Gegenständen kam es bei Köchen signifikant häufiger zu Unfällen. Messer waren bei Köchen doppelt so häufig am Unfallgeschehen beteiligt wie bei Küchenhilfen (34\% vs. 16,1\%).

Die Unfälle in der Gruppe der Küchenhilfen lassen sich signifikant häufiger auf das Stolpern bei gehender Bewegung zurückführen. Küchenhilfen waren häufiger von Unfällen betroffen, die sich beim manuellen Heben, Schieben oder Ziehen von Gegenständen ereigneten $(p<0,01)$, die auf eine akute körperliche Überlastung des Bewegungsapparates zurückzuführen waren oder sich beim Aufprallen gegen ortsfeste Gegenstände ereignet hatten. Dazu passt, dass es sich bei den am Unfall beteiligten Gegenständen häufiger bei Küchenhilfen um bauliche Einrichtungen handelte, wobei Fußböden am häufigsten beteiligt waren. Die Beschaffenheit des Untergrunds - wie rutschige Fußböden infolge von Wasser oder Fettresten - war für weniger als $5 \%$ der Unfälle verantwortlich.

\section{BK-Geschehen}

Von insgesamt 2340 meldepflichtigen Verdachtsanzeigen machten Hauterkrankungen mit über $80 \%$ den größten Teil aus ( $\mathbf{0}$ Tab. 2). An zweiter und dritter Stelle folgten bandscheibenbedingte Erkrankungen der Lendenwirbelsäule und obstruktive Atemwegserkrankungen. Infektionskrankheiten oder Beeinträchtigung des Hörvermögens aufgrund von Lärm spielten für die hier betrachteten Berufsgruppen nur eine untergeordnete Rolle. Hauterkrankungen wurden häufiger von Küchenhilfen gemeldet, bandscheibenbedingte Erkrankungen der Lendenwirbelsäule (BK 2108) dagegen häufiger von Köchen. 
Tab. 2 Meldepflichtige Verdachtsanzeigen auf eine Berufskrankheit nach Krankheitsgruppe

und Berufsgruppe-Daten der Berufsgenossenschaft für Gesundheitsdienst und Wohlfahrtspflege (BGW) für 2008 bis 2017

\begin{tabular}{|c|c|c|c|c|c|c|}
\hline \multirow[t]{3}{*}{ Krankheitsgruppe } & \multicolumn{6}{|c|}{ Berufsgruppe } \\
\hline & \multicolumn{2}{|c|}{$\begin{array}{l}\text { Küchenhilfen } \\
n=1660^{\mathrm{a}}\end{array}$} & \multicolumn{2}{|c|}{$\begin{array}{l}\text { Köche } \\
n=680^{\mathrm{a}}\end{array}$} & \multicolumn{2}{|c|}{$\begin{array}{l}\text { Gesamt } \\
n=2340^{\mathrm{a}}\end{array}$} \\
\hline & $n$ & $\%$ & $n$ & $\%$ & $N$ & $\%$ \\
\hline Hauterkrankungen (BK 5101) & 1453 & 87,5 & 553 & 81,3 & 2006 & 85,7 \\
\hline $\begin{array}{l}\text { Physikalische Einwirkungen } \\
\text { Darunter: }\end{array}$ & 124 & 7,5 & 77 & 11,3 & 201 & 8,6 \\
\hline $\begin{array}{l}\text { Erkrankungen der Sehnenscheiden } \\
\text { (BK 2101) }\end{array}$ & 6 & - & 6 & - & 12 & - \\
\hline $\begin{array}{l}\text { Bandscheibenbedingte Erkrankun- } \\
\text { gen der Lendenwirbelsäule (BK } \\
\text { 2108) }\end{array}$ & 74 & - & 49 & - & 123 & - \\
\hline $\begin{array}{l}\text { Bandscheibenbedingte Erkrankun- } \\
\text { gen der Halswirbelsäule (BK 2109) }\end{array}$ & 11 & - & 6 & - & 17 & - \\
\hline Karpaltunnel-Syndrom (BK 2113) & 7 & - & 3 & - & 10 & - \\
\hline Lärmschwerhörigkeit (BK 2301) & 18 & - & 6 & - & 24 & - \\
\hline $\begin{array}{l}\text { Erkrankungen der Atemwege und } \\
\text { Lungen } \\
\text { Darunter: }\end{array}$ & 40 & 2,4 & 29 & 4,3 & 69 & 2,9 \\
\hline $\begin{array}{l}\text { Obstruktive Atemwegserkrankun- } \\
\text { gen }\end{array}$ & 38 & - & 27 & - & 65 & - \\
\hline Infektionskrankheiten (BK 3101) & 23 & 1,4 & 5 & 0,7 & 28 & 1,2 \\
\hline Sonstige $e^{a}$ & 20 & 1,2 & 16 & 2,4 & 36 & 1,5 \\
\hline
\end{tabular}

Bei 2,3\% der Fälle mit bestätigtem BK-Verdacht wurde die Erkrankung als BK anerkannt (44 von 1880). Bei den übrigen bestätigten Fällen waren die besonderen versicherungsrechtlichen Voraussetzungen nicht erfüllt, da die Beschäftigten ihre Tätigkeit weiter ausüben konnten. In 98,2 \% der bestätigten Fälle handelte es sich um Hauterkrankungen, für die Kontakt mit Wasser, Desinfektions- und Reinigungsmitteln oder Lebensmitteln die häufigsten Auslöser waren (•Tab. 3). Atemwegserkrankungen waren in 12 von 19 Fällen durch Lebensmittel ausgelöst. Auf Infektionserreger waren 11 Fälle zurückzuführen und auf Belastungen durch Spülmaschinenlärm oder extreme Rumpfbeugung jeweils 1 Fall.

\section{Diskussion}

Für die vorliegende Arbeit wurden Routinedaten zum Arbeitsunfall- und BKGeschehen bei Küchenhilfen und Köchen ausgewertet. Für beide Gruppen zeigten sich zwei unterschiedliche Un- fallmuster: Küchenhilfen erlitten signifikant häufiger Stolperunfälle und verletzten sich häufiger durch starke körperliche Belastung z. B. beim manuellen Transport von Gegenständen oder Prallen gegen Gegenstände. Köche dagegen verletzten sich signifikant häufiger beim Umgang mit spitzen, scharfen Gegenständen, durch Verlust der Kontrolle über handgeführte Gegenstände oder durch Verbrennungen. BK-Meldungen zu Hauterkrankungen dominierten mit einem Anteil von über $80 \%$ das BK-Geschehen in beiden Gruppen und wurden am häufigsten durch Kontakt zu Wasser und Reinigungs- oder Desinfektionsmittel ausgelöst.

Beschäftigte in Küchenbetrieben sind eher selten die Zielgruppe in arbeitsmedizinischen Studien. Die meisten Studien wurden zu muskuloskeletalen Beschwerden und Schnittverletzungen sowie Verbrennungen durchgeführt [5-7]. Muskuloskeletale Beschwerden hatten bei taiwanesischen Köchen eine jährliche Inzidenz von $25 \%$ und bei finnischen, weiblichen Küchenhilfen eine 3-Monats-Präva- lenz für Beschwerden in Nacken-, Schulter- und Handbereich von $71 \%$, 34\% bzw. $49 \%[7,14]$. In der von uns ausgewerteten Stichprobe bezogen sich 7,8\% der Verdachtsanzeigen auf Erkrankungen des Bewegungsapparats (BK-Ziffern 2101 bis 2113). Eine Auswertung von Routinedaten der nationalen Krankenversicherung Taiwans ergab, dass die berufliche Tätigkeit als Koch einen Risikofaktor für Sehnenscheidenentzündung darstellt (Odds-Ratio 2,52; [14]). In den uns vorliegenden Daten findet sich hierfür kein Beleg.

In unserer Stichprobe aus Küchenhilfen und Köchen handelte es sich bei fast $98 \%$ der bestätigten BK-Fälle um Hauterkrankungen. Im Vergleich $\mathrm{zu}$ anderen Berufsgruppen lag der Anteil an Hauterkrankungen bei Küchenpersonal in klinischen und pflegerischen Einrichtungen damit deutlich über dem der übrigen UV-Träger (durchschnittlich 59,7\% im Jahr 2018; [13]). Tätigkeiten in Küchenbetrieben werden von vielen Autoren als hautbelastend eingestuft, allerdings finden sich nur wenige Angaben zur Prävalenz von Hauterkrankungen in den betroffenen Berufsgruppen. Bei indischem Küchenpersonal lag die Prävalenz von Handekzemen bei $10 \%$ [9]. Der hohe Anteil von Hauterkrankungen am BKGeschehen in Deutschland ist vermutlich darauf zurückzuführen, dass nach Einführung des Hautarztverfahrens im Jahr 2006, die damit verbundenen $\$ 3$-Maßnahmen als bestätigte Berufskrankheiten statistisch erfasst werden. Die beruflichen Konsequenzen von Handekzemen wurden in einer Kohortenstudie u. a. auch bei Küchenpersonal untersucht [11]. Danach hatten $54 \%$ der Beschäftigten in Küchenbetrieben 7 bis 14 Jahre nach der Diagnose des Handekzems ihre berufliche Tätigkeit gewechselt gegenüber durchschnittlich $34 \%$.

In einer japanischen Studie zu Einflussfaktoren auf Nahrungsmittelallergien wurden chronische Handekzeme als ein bedeutender Risikofaktor bei Beschäftigten mit Küchenarbeit identifiziert verglichen mit Beschäftigten ohne Küchenarbeit (Odds-Ratio 2,4; [12]). Die Autoren führen dies auf den häufigen Kontakt mit Fisch- und Fruchtsäuren zurück. Für chronisch-obstruktive Atem- 
Tab. 3 BK-auslösender Gegenstand bei Beschäftigten in Küchenbetrieben $(n=1820)^{\mathrm{a}}$ - Daten der Berufsgenossenschaft für Gesundheitsdienst und Wohlfahrtspflege (BGW) für 2008-2017 Krankheitsgruppe mit BK auslösendem Gegenstand

\begin{tabular}{|c|c|c|c|}
\hline Gegenstand & Anzahl & $\begin{array}{l}\text { \% an } \\
\text { Gesamt }\end{array}$ & $\begin{array}{l}\text { \% an Krank- } \\
\text { heitsgruppe }\end{array}$ \\
\hline Hautkrankheiten & 1787 & 98,2 & - \\
\hline Wasser, Feuchtmilieu (z. B. durch Handschuhtragen) & 793 & - & 44,4 \\
\hline $\begin{array}{l}\text { Desinfektionsmittel, Konservierungsmittel, Duft- } \\
\text { stoffe }\end{array}$ & 597 & - & 33,4 \\
\hline Reinigungs-/Spülmittel & 249 & - & 13,9 \\
\hline Lebensmittel/-Staub & 59 & - & 3,3 \\
\hline Gummi-Inhaltsstoffe/Chemische Substanzen ${ }^{\text {b }}$ & 89 & - & 5,0 \\
\hline Allergische und toxische Atemwegserkrankungen & 19 & 1,0 & - \\
\hline Lebensmittel/-Staub & 12 & - & 63,2 \\
\hline Desinfektionsmittel & 5 & - & 26,3 \\
\hline Duftstoff & 1 & - & 5,3 \\
\hline Formaldehyd & 1 & - & 5,3 \\
\hline Infektionskrankheiten & 11 & 0,6 & - \\
\hline Mykobakterien & 6 & - & 54,5 \\
\hline Parasiten z. B. Krätzmilben, Läuse & 5 & - & 45,5 \\
\hline $\begin{array}{l}\text { Bandscheibenbedingte Erkrankungen der Lendenwir- } \\
\text { belsäule: extreme Rumpfbeugung }\end{array}$ & 1 & $<0,1$ & 100,0 \\
\hline Lärm:Spülmaschine & 1 & $<0,1$ & 100,0 \\
\hline Kohlenmonoxid & 1 & $<0,1$ & 100,0 \\
\hline
\end{tabular}

wegserkrankungen und Infektionen der oberen Atemwege wurden 1,3- bis 1,6fach erhöhte Odds-Ratios für Beschäftigte in Küchenbetrieben ermittelt, wofür neben hohen Wärme- und Feuchtlasten auch Fettaerosole in Kochdämpfen verantwortlich gemacht wurden $[1,2]$. In der uns vorliegenden Stichprobe war der Anteil an Atemwegserkrankungen an allen bestätigten BK-Fällen vergleichbar mit dem der übrigen UV-Träger (durchschnittlich 1,9\%; [13]).

Schnittverletzungen als die häufigsten Arbeitsunfälle von Köchen werden auch von anderen Autoren beschrieben $[5,6]$. Psychischer und physischer Stress im beruflichen Alltag waren wichtige Risikofaktoren für Schnittverletzungen und Verbrennungen unter Beschäftigten in Küchenbetrieben (Odds-Ratio zwischen 2,2 und 4,5; [6]). Über einen hohen Anteil an Stolperunfällen unter Küchenpersonal berichteten verschiedene Autoren [17]. In der hier untersuchten Stichprobe war jeder vierte Unfall ein Stolper-, Rutsch- oder Sturzunfall, was dem Anteil entspricht, den diese Unfälle am Unfall- geschehen bei den übrigen UV-Trägern im Jahr 2015 hatten (21 \%) [4]. Rutschunfälle werden häufig in Kühlräumen und Spülküche beobachtet und oft im Zusammenhang mit feuchten oder fettigen Fußböden [16]. In der hier untersuchten Stichprobe spielten rutschige Böden nur eine untergeordnete Rolle.

Zur Vermeidung der vielfältigen Gefährdungen im Küchenbereich sind in der DGUV-Regel 110-003 grundlegende Präventionsmaßnahmen beschrieben [3]. Speziell für den Bereich der Spülküche wurden ergonomische Gestaltungsmaßnahmen zusammengetragen [8]. Für Beschäftigte in Küchenbetrieben mit Verdacht auf eine beruflich bedingte Hauterkrankung werden im Rahmen des Hautarztverfahrens von verschiedenen UV-Trägern Hautschutzseminare mit branchenbezogenen Präventionsangeboten angeboten, um einen Verbleib im Berufs zu ermöglichen [15, 18].

Für diese Studie gibt es einige Limitationen. 1. Durch Verwendung der BGWDatenbanklassen sich die Ergebnisse ausschließlich auf Beschäftigte in Einrich- tungen des Gesundheitsdienstes und der Wohlfahrtspflege übertragen. 2. Die mit Routinedaten verbundenen Einschränkungen betreffen auch die vorliegende Auswertung: Die Erfassung der Routinedaten unterliegt Vorgaben der DGUV zur Dokumentation des Unfall- und BKGeschehens und erfolgt nicht primär für wissenschaftliche Zwecke, sondern für die Steuerung des Heilverfahrens. Unterschiede zwischen unseren Ergebnissen zur Häufigkeit von muskuloskeletalen Erkrankungen und den zitierten Studien führen wir auf die unterschiedlichen Datenquellen zurück: Die Fälle aus den Routinedaten der Unfallversicherung erfüllen die rechtlichen Voraussetzungen für die Anerkennung bzw. Bestätigung des BK-Verdachts. Die Ergebnisse der zitierten Studien basieren dagegen auf Eigenangaben von Teilnehmern in Studien zu gesundheitlichen Beschwerden.

\section{Fazit für die Praxis}

Hauterkrankungen, Stolperunfälle und Schnittverletzungen dominieren das Unfallgeschehen in Küchenbetrieben. Der hohe Anteil an Feuchtarbeit in Küchenbetrieben erfordert die konsequente Anwendung von Hautschutzmaßnahmen. Die Unfall- und BK-Daten bieten eine gute Datenlage für zielgruppenbezogene Präventionsmaßnahmen für Beschäftigte in Küchenbetrieben. Die Präventionsmaßnahmen zur Vermeidung dieser Berufskrankheiten und Unfälle sollten weiterentwickelt bzw. auf ihre Effektivität hin überprüft werden.

\section{Korrespondenzadresse}

\section{Dr. Madeleine Dulon, MPH}

Abteilung Arbeitsmedizin, Gefahrstoffe, Gesundheitswissenschaften, Berufsgenossenschaft für Gesundheitsdienst und Wohlfahrtspflege

Pappelallee 33-37, 22089 Hamburg,

Deutschland

madeleine.dulon@bgw-online.de

\section{Einhaltung ethischer Richtlinien}

Interessenkonflikt. M. Dulon, D. Wendeler, C. Westermann und A. Nienhaus geben an, dass kein Interessenkonflikt besteht. 
Für diesen Beitrag wurden von den Autoren keine Studien an Menschen oder Tieren durchgeführt. Für die aufgeführten Studien gelten die jeweils dort angegebenen ethischen Richtlinien.

Open Access Dieser Artikel wird unter der Creative Commons Namensnennung 4.0 International Lizenz veröffentlicht, welche die Nutzung, Vervielfältigung, Bearbeitung, Verbreitung und Wiedergabe in jeglichem Medium und Format erlaubt, sofern Sie den/die ursprünglichen Autor(en) und die Quelle ordnungsgemäß nennen, einen Link zur Creative Commons Lizenz beifügen und angeben, ob Änderungen vorgenommen wurden.

Die in diesem Artikel enthaltenen Bilder und sonstiges Drittmaterial unterliegen ebenfalls der genannten Creative Commons Lizenz, sofern sich aus der Abbildungslegende nichts anderes ergibt. Sofern das betreffende Material nicht unter der genannten Creative Commons Lizenz steht und die betreffende Handlung nicht nach gesetzlichen Vorschriften erlaubt ist, ist für die oben aufgeführten Weiterverwendungen des Materials die Einwilligung des jeweiligen Rechteinhabers einzuholen.

Weitere Details zur Lizenz entnehmen Sie bitte de Lizenzinformation auf http://creativecommons.org/ licenses/by/4.0/deed.de.

\section{Literatur}

1. Bilge U, Unluoglu I, Son N et al (2013) Occupational allergic diseases in kitchen and health careworkers: an underestimated health issue. Biomed Res Int 2013:285420

2. De Matteis $S$, Jarvis D, Hutchings $S$ et al (2016) Occupations associated with COPD risk in the large population-based UK biobankcohortstudy. Occup Environ Med 73:378-384

3. DGUV (2019) Branche Küchenbetriebe - DGUV Regel 110-003. https://publikationen.dguv. de/regelwerk/regelwerk-nach-fachbereich/ nahrungsmittel/gastgewerbe/1338/branchekuechenbetriebe.Zugegriffen:4.Nov. 2019

4. DGUV (2017) Fact Sheet Stolper-, Rutsch-und Sturzunfälle. https://www.dguv.de/medien/inhalt/ mediencenter/pm/pressearchiv/2017/1_quartal/ factsheet_srs_au.pdf.Zugegriffen: 19.Sept. 2019

5. Gleeson D (2001) Health and safety in the catering industry. Occup Med 51:385-391

6. Haruyama Y, Matsuzuki H, Tomita S et al (2014) Burn and cut injuries related to job stress among kitchen workers in Japan. Ind Health 52:113-120

7. Haukka E, Leino-Arjas P, Solovieva Set al (2006) Cooccurrence of musculoskeletal pain among female kitchen workers. Int Arch Occup Environ Health 80:141-148

8. Heinicke K, Friedrichsen G, Heidl H et al (2000) Ergonomische Bewertung der Arbeitsbedingungen in einer Spülküche und Gestaltungsmaßnahmen. https://publikationen.sachsen.de/bdb/artikel/ 14871.Zugegriffen:4. Nov. 2019

9. Kokane S, Tiwari RR (2011) Occupational health problems of highway restaurant workers of Pune, India. Toxicol Ind Health 27:945-948

10. Lund T, Flachs EM, Ebbehoj NE et al (2019) Wet work exposure: comparison of observed and selfreported data. Int Arch Occup Environ Health 92:317-326

11. Malkönen T, Alanko K, Jolanki R et al (2010) Long-term follow-up study of occupational hand eczema. Br J Dermatol 163:999-1006
12. Minami T, Fukutomi Y, Sekiya K et al (2018) Hand eczema as a risk factor for food allergy among occupational kitchen workers. Allergol Int 67:217-224

13. Schwabbacher W, Meier K, Forsch K (2019) Kennziffern derDeutschen Gesetzlichen Unfallversicherung 2018. DGUV Forum, S40-55

14. Shiue HS, Lu CW, Chen CJ et al (2008) Musculoskeletal disorder among 52,261 Chinese restaurant cooks cohort: result from the National Health Insurance Data.J Occup Health 50:163-168

15. Soder S, Diepgen TL, Radulescu M et al (2007) Berufsbedingte Hauterkrankungen bei Beschäftigten in Reinigungs- und Küchenberufen: Verlauf und Lebensqualität nach Maßnahmen der Sekundären Individualprävention. J Dtsch Dermatol Ges 8:670-677

16. Varnai A, Nienhaus A, Groneberg DA et al (2018) Körperhaltungen von Arbeitnehmern am Beispiel einer Krankenhausgroßküche. Eine objektive Tätigkeitsanalyse. Zentralbl Arbeitsmed 68:197-205

17. Verma SK, Chang WR, Courtney TK et al (2010) Workers' experience of slipping in U.S. limitedservice restaurants. JOccup Environ Hyg 7:491-500

18. Weisshaar E, Radulescu M, Soder S et al (2007) Secondary individual prevention of occupational skin diseases in health care workers, cleaners and kitchen employees: aims, experiences and descriptive results. Int Arch Occup Environ Health 80:477-484 